\title{
Active case-finding and enhanced data collection to identify neglected tropical diseases
}

\author{
Nhlanhla Nhlabatsi ${ }^{\star 1}$, Vusie Lokotfwako', Dumsile Mabundza', Fortunate Bhembe ${ }^{1}$, \\ Phinda Khumalo', Njabulo Lukhele', Elizabeth Mvila1', Siphiwe M. Shongwe-Gama², \\ Thulani Maphosa ${ }^{2}$ and Harriet Nuwagaba-Biribonwoha ${ }^{2}$
}

${ }^{1}$ Ministry of Health, Mbabane, Swaziland; ${ }^{2}$ ICAP Columbia University, Mbabane, Swaziland

\section{Objective}

To strengthen public health surveillance to monitor neglected tropical diseases (NTDs) like leprosy as a control measure to avert disabilities in the Kingdom of Swaziland.

\section{Introduction}

Leprosy is a chronic infectious disease caused by Mycobacterium leprae. It is a contagious disease that affects the skin, mucous membrane, and nerves causing discoloration and lumps on the skin and in severe cases disfigurement and deformities. The mode of transmission remains uncertain, but is believed that M.leprae is spread from person to person primarily as a nasal droplet infection. The incubation period for a bacterial disease generally is $5-7$ years. Progress in the fight against leprosy has been one of the greatest public health success and in the country, was eliminated in the mid1990s. However on the $22^{\text {nd }}$ August 2017 a confirmed leprosy cases was reported by the National Referral Hospital.

\section{Methods}

Following the confirmed case an investigation was conducted to fully understand the possible source of the case and identify further cases. The assessment was done in three parts that is, hospital visit to follow up on the index case; conducting home visits to collect data for leprosy and their contact and a file review of all clients who were once diagnosed as having leprosy

\section{Results}

The index case was identified and his condition is improving as he has been initiated on MDT which he will be taking for a minimum of six months. Eleven clients were visited in their homes. Their age range was 31 to 91 years but the majority were above 60 years with a median age of 70 years. There were 7 females and 4 males.. The clients presented with permanent nerve damage either from the face, upper or lower limbs. The common disabilities and deformities post treatment were sagging of face, nasal collapse, blindness and clawing of fingers and feet. Other patients had plantar-palmar ulcers and abscesses from trauma, injuries or burns sustained due to nerve damage and inadequate protection. They reported to be experiencing stigma and are being discriminated. None of the clients presented with clinical signs and symptoms suggestive of leprosy. There were 18 files that were reviewed out of 58 who are known to exist. Six of the 18 files belonged to clients who were seen during home visits. One of the clients was epidemiologically linked to the index case as they used to live together in 1994.

\section{Conclusions}

The country seem to be experiencing the re-emerging of leprosy. Since the index case is epidemiologically linked to one of the old cases this therefore confirms the incubation period of leprosy being from 15 to 20 years. There is need to strengthen leprosy prevention and control measures as well as strengthening of leprosy surveillance in the context of IDSR. There is an urgent need to raise public awareness and provide clients with protective clothing. Furthermore, there is need to strengthen the Bilharzia and Worms Control Program to incorporate leprosy as it is one of the NTDs targeted for elimination in Swaziland.

Keywords

leprosy; case-finding; Swaziland

\section{Acknowledgments}

1. Ministry of Health, Mbabane, Swaziland

2. ICAP, Columbia University, Mailman School of Public Health, Mbabane, Swaziland

3. Columbia University, Department of Epidemiology, Mailman School of Public Health, New York, United States of America

\section{References}

1. Heymann D.L 2008, Control of Communicable Diseases Manual, $18^{\text {th }}$ Edition, American Public Health Association, Geneva, Switzerland.

*Nhlanhla Nhlabatsi

E-mail: ntini0064@gmail.com 